\title{
Both classical \& quantum information: both bit \& qubit. Both physical \& transcendental time
}

\author{
Vasil Penchev, vasildinev@gmail.com \\ Bulgarian Academy of Sciences: Institute of Philosophy and Sociology: \\ Dept. of Philosophy of Science
}

\begin{abstract}
Information can be considered as the most fundamental, philosophical, physical and mathematical concept originating from the totality by means of physical and mathematical transcendentalism (the counterpart of philosophical transcendentalism). Classical and quantum information, particularly by their units, bit and qubit, correspond and unify the finite and infinite. As classical information is relevant to finite series and sets, as quantum information, to infinite ones. A fundamental joint relativity of the finite and infinite, of the external and internal is to be investigated. The corresponding invariance is able to define physical action and its quantity only on the basis of information and especially: on the relativity of classical and quantum information. The concept of transcendental time, an epoché in relation to the direction of time arrow can be defined. Its correlate is that information invariant to the finite and infinite, therefore unifying both classical and quantum information.
\end{abstract}

Key words: bit and qubit, classical and quantum information, epoché, physical and mathematical transcendentalism, qubit space (qubit Hilbert space), separable complex Hilbert space, the totality, transcendental time

\section{HISTORICAL BACKGROUND}

One can trace the pathway from classical to quantum information:

Information was defined initially (Shannon 1948) as a quantity (thus a real number in general) referring to encoding or transferring data in technical systems. It can be represented (e.g.) as the number of choices corresponding to a certain number of alternatives after the process of encoding, therefore being at the same time a quantity of the useful knowledge transferred and processed in technical systems.

In the same 1948, Norbert Wiener proclaimed the new science of cybernetics (a neologism coined by Wiener) studying the control and communication in animals and machines implicitly representing informational processes ${ }^{1}$. The fruitful idea was generalized very soon to any biological, technical and even social systems as well as to systems at all, in the framework of so-called systemology. Whatever a system is, it transfers and processes information, the same in any system and shares the same informational laws expressly mathematically. Nowadays, cybernetics results in contemporary computer science, cognitive science, neuroscience, etc.

The new science suggested by Wiener is able to consider information as a kind of substance (at least conventionally) able to unify technical, biological, social, and abstract

\footnotetext{
${ }^{1}$ Wiener $(1950 ; 1964)$ generalized his ideas furthermore in relation to society and religion.
} 
systems considering any certain one as a kind of exemplification of what a system is. Cybernetics relates the concept of "information" to those of "control", "communication", and "system". All the four can be represented abstractly and mathematically, thus implying a mathematical theory of information, particularly. Nonetheless, cybernetics means information only in relation to real systems whether technical or whatever and restricts information to that of finite sets or series, as to its mathematical formalism.

A few papers of Kolmogorov $(1965 ; 1968)$ researched a few generalizations once information had been defined as a certain function (as Shannon did) by the direct generalization of that function to a wider class of functions, to which it is a representative or a particular case:

Information was connected to another fundamental concept, probability (respectively, probability distribution, probability density distribution, etc.). Furthermore, Kolmogorov suggested a non-conservative generalization of information, the so-called today "Kolmogorov complexity" (Kolmogorov 1963; 1998; Martin-Löf 1966) meaning the information of a constructive series able to generate a system abstractly and mathematically modelled.

The definition of information is isomorphic (eventually under an elementary additional condition such as anti-isometry) to the quantity of entropy in thermodynamics referring to statistical ensembles. Information as "negentropy" can be defined even only in terms of set theory as referring to the probability distributions of the elements of a class of equivalence. The consideration of information as entropy (or vice versa: that of entropy as information) suggests that the ideas of cybernetics can be extended even further: to the physical cognition and world.

The unprecedented development of computers underlain by the processing of information deforms the initial intention of cybernetics to a rather technical concept though fundamental as to computer science, but only to it. Information lost its just acquired status of a unifying interdisciplinary conception therefore preventing any further generalization to a philosophical notion or category. However, the downward trend was broken by the idea of "quantum computer" processing "quantum information" furthermore able to reformulate quantum mechanics (the most fundamental physical theory) as an information theory thoroughly and absolutely.

Quantum mechanics realizes that extension of cybernetic ideas especially radically: by reformulating itself as an information theory absolutely and thoroughly, namely the theory of quantum information. Quantum information can be considered as the free variable, any value of which is a wave function, as to terms of quantum mechanics properly. Furthermore, quantum information can be defined as that generalization of classical information referring to infinite series or sets (unlike the classical information itself referring to finite ones).

Quantum mechanics states that any physical system is quantum in the final analysis, but non-quantum and classical only approximately, in relation to physical action much, much greater than the fundamental Planck constant. Then, any physical system is a quantum-information system, and quantum information is the universal general omnipresent substance of the physical, "bodily", and empirical world. Furthermore, any quantum state, 
and thus, also any quantum-information state is the same regardless of being considered whether as physical, "bodily" or as cognitive, "mental" therefore bridging the both sides of dualistic abyss just as the philosophical category of "Time" did and does.

\section{THE UNITS OF INFORMATION}

A bit of information is usually defined as an elementary choice between two equally probable alternatives. This is furthermore the least possible amount of information if it is defined as Shannon did (i.e. analogically to physical entropy). Mathematically, this means the equivalence of the ordering of two elements (being well-ordered always) and the choice among a set consisting of two elements. A bit of information can be visualized as a record of either " 0 " or " 1 " in a tape cell of a Turing machine, being "empty" in advance.

The word "qubit" is a neologism, abbreviation from "quantum bit" = qubit, i.e. literally a "unit of quantum information". It is initially defined by means of the separable complex Hilbert space utilized by quantum mechanics as the normalized superposition of two orthogonal subspaces ${ }^{2}$ of that Hilbert space. Particularly, the separable complex Hilbert space itself can be represented equivalently as the so-called qubit Hilbert space since any two successive axes of it (i.e. $e^{i n \omega}$ and $e^{i(n+1) \omega}$ ) are two orthogonal subspaces of that Hilbert space and can constitute a qubit.

Any element of the separable complex Hilbert space being a series or a set of complex numbers can be equivalently defined by pairs of complex numbers furthermore normed, each of which constitutes a qubit. Then, any element of the qubit Hilbert space will be a series or a set of qubits. That Hilbert space is also anti-isometric to its dual space and keeps unitarity interpretable physically as energy conservation. It can be granted as isomorphic to the standard complex Hilbert space (under inessential additional conditions), but with the advantage for all physical processes to be interpretable as informational.

The cited definition of "qubit" in quantum mechanics and information can be represented equivalently as that generalization of a bit which can be referred to the information of infinite series and sets (Penchev 2020 July 10). If one considers infinite series or sets, the quantity of classical information measured in the corresponding units of bits is arbitrary, being always "infinite". Thus, the quantity of information in any infinite series or set needs special units turning out to be equivalent to the units of quantum information, qubits.

Then, "qubit" as it is defined in quantum mechanics can be considered as that generalization of "bit", which is to be related to infinite sets and series. Bits and qubits are both embedded in the qubit Hilbert space as follows:

Any pair of dual qubits enumerated by the same natural number constitutes a bit. The set of all bits delivered as above constitutes a qubit in turn. Interpreted so, the qubit Hilbert space consists of all states of itself (e.g. in each qubit separately, but not only).

Any wave function is equivalent to a value as a value of quantum information, but being interpreted initially in quantum mechanics as a state of the quantum system. Thus, any value of quantum information is a state of the quantum system, i.e. relevant to a physical phenomenon in the final analysis.

\footnotetext{
${ }^{2}$ In fact, those orthogonal subspaces of Hilbert space can be as convex as nonconvex to each other.
} 
The value of quantum information can be interpreted furthermore purely mathematically, even arithmetically (in a generalized sense discussed further) as a transfinite natural number. As any transfinite ordinal number is interpretable as a state of infinity, both can be thought as a quantum state not less, but by the mediation of quantum information.

Physics and mathematics seem to be absolutely different even in their bases: experimental and postulative correspondingly. Nonetheless, they have turned out to be linked closely since the dawn of modern physics, since Galileo and Newton though opposed in virtue of Cartesian doctrine: correspondingly, on the side of "body" versus that of "mind" in Descartes's dualism. Wave function overcomes that gap because it being the same is "bodily" in physics (as a quantum state) and "mental" in mathematics (as a transfinite ordinal or natural number) therefore unifying their foundations.

As far as any physical system is quantum (and classical whether only approximately or complimentary), wave function is fundamental: this is commonly accepted. However, the statement that it being an element in an as derivative structure as the separable complex Hilbert space is fundamental nor less as to mathematics than to physics is extraordinary, even scandalous. Its main reason is the similarity or even equivalence of the consistent completeness of quantum mechanics (proved by the theorems of the absence of hidden variables in quantum mechanics), on the one hand, and the problematic consistent completeness of mathematics (after the Gödel incompleteness theorems), on the other hand (Penchev 2020 August 25).

The Cartesian episteme of Modernity postulates an abyss between the "bodily" (empirical and experimental) physics and the "mental" (axiomatic and deductive) mathematics. However, only the physical quantity of time or the philosophical category of Time are able to bridge the abyss, but only sometimes.

On the contrary, information unifies them in definition. Furthermore, it is able to include all being temporal or even to admit the being of non-temporal entities. So, information transforms the transition between physics and mathematics to be gradual, continuous and smooth.

The ancient Pythagoreanism had been postulated for the foundation of the world to be arithmetical and thus mathematical in the final analysis.

Nowadays, quantum mechanics was forced to partly restore the worldview of Pythagoreanism as far as the gap of mathematics and physics was necessary to be complemented by a smooth transition between them. That necessity can be proved even only within the formalism of quantum mechanics as the theorems of the absence of hidden variables (Neumann 1932; Kochen, Specker 1967). Information being both physical and mathematical concepts is able to realize the unification of physics and mathematics in their foundations.

The approach of quantum mechanics can be traced stull to the statistic (mathematical in fact) interpretation of phenomenological (i.e. physical) interpretation:

The initial "phenomenological" thermodynamics is predetermined for itself to be defined only on a macroscopic level. Statistical thermodynamics complements it with another, hidden level: microscopic and actual in Boltzmann's, but macroscopic and virtual in Gibbs's. 
Entropy (or the specific class of similar quantities to which it belongs) in statistical thermodynamics is that quantity able to connect the two levels; and more precisely: it represents the phenomenological level of experience referring to a whole as a function of the other, hidden (and eventually only postulated) level.

One might introduce an auxiliary quantity to any finite set (and generalizable to infinite ones) meaning the number of partial different orderings, each of which being well-ordering, but incommensurable with any of all the rest. The greater is that auxiliary quantity, the bigger is the abstract entropy of the set at issue and represents the quantitative degree of its disordering (respectively and from the opposed viewpoint: ordering). That mathematical entropy and physical entropy share the pair of two disjunctive levels, however interpreted differently: the whole of the set versus that of its elements, as to mathematical entropy.

The pair of entropy and information is equally applicable to physical states as to mathematical ones. If one notices that entropy is more met in physics, the reason might be that the physicists are "pessimists" unlike the "optimistic" mathematicians ${ }^{3}$. Or in dualistic terms: the bodily subject of physics is disordered by itself unlike the mental subject of mathematics ordered by the mathematicians themselves. Nonetheless, both mean the same.

\section{SCIENTIFIC TRANSCENDENTALISM}

The usual "philosophical transcendentalism" is granted to be unfalsifiable, i.e. a properly philosophical doctrine. Thus, it is included in the "eternal" treasure of the most valuable human ideas therefore protected from refutation "forever" regardless of the historically restricted scope of scientific knowledge or possible cognition in any epoch.

On the contrary, its counterpart of scientific transcendentalism would be to formulate its theses in a way falsifiable by experience and experiment just in a certain historical episteme, to which those formulations make sense.

In fact, one needs a general and scientific definition of the totality comprising the particular examples of its uses in different sciences. Those exemplifications might be: the universe, system, biological organism, chemical compound, physical entity, society, neural network, etc. So, scientific transcendentalism would imply a unifying and interdisciplinary approach applicable to astronomy and astrophysics, systemology, biology, chemistry, physics, sociology, history, neuroscience, etc.

Being "all" in definition, the totality should contain its externality within itself by itself. So, it generates definitively a fundamental and initial doubling of anything; once it by itself and twice: again it, but now as a representative of its external counterpart. The formal, both logical and mathematical structure is that of a bit of information. Any doubling of the above kind (which can be called "transcendental doubling") consists of itself and two alternatives: "inside" and "outside" of the totality.

\footnotetext{
${ }^{3}$ One may coin the humorous metaphor of entropy as the pessimist's information (respectively, information as the optimist's entropy). Both optimists and pessimists observe the same partly ordered, partly disordered state, but the former interprets it as ordered by information, and the latter as disordered by entropy.
} 
Information (not being yet "transcendental") can be defined as that quantity "unitable" (or measurable) by units of bits. Then, what is unitable by elementary doublings of anything with its external counterpart will be called "transcendental information". An elementary doubling can be defined in turn and independently as a generalized identity after the identification of anything with its external counterpart after transcendental doubling. Furthermore, that definition of "elementary doubling" is to be postulated as an equivalent of a bit of information, after which both alternatives are equally probable.

A bit of information implies irreversibility following the direction of choice of any disjunctive alternative. So, any transcendental doubling implies a corresponding irreversibility: from the doubling itself to the alternative either within the totality or its counterpart out of the totality. The same irreversibility implies furthermore just one reversibility if it is unified with the same irreversibility, but "running backwards": from the distinction of both alternatives (either within or out of the totality) back to the coherent state of the doubling itself.

As to irreversibility (regardless of which direction), the alternatives and their unification as the doubling themselves are ordered, even well-ordered, being a natural number. As to reversibility, they are not being "simultaneous". One can postulate as obvious (as an "axiom") that both irreversibilities are equivalent to the reversibility therefore falling in turn into the formal structure of a bit of information. As to an infinite set of alternatives, the analogical statement is much less obvious, but nonetheless it can be granted to be true as "transcendental invariance", furthermore provable ${ }^{4}$ in set theory as the equivalence of the axiom of choice and the well-ordering "theorem".

One can define each of both irreversibilities as an elementary irreversibility. Then, if one grants any elementary irreversibility as a unity of the quantity of "transcendental time", the transcendental time can be defined as that quantity "unitable", i.e. measureable by the units of elementary irreversibility. So in the final analysis, the ultimate conclusion is: scientific transcendentalism is able to generate two fundamental "transcendental" quantities: time and information.

"Time", at least since Heidegger's "Sein und Zeit" (1927), is one established philosophical category: a speculative and metaphysical counterpart of our temporal experience or the physical quantity of time. Furthermore, "Time" is able to unify the two sides of the dualistic Cartesian abyss because it is shared by both "Mind" and "Body" (unlike "Space" situated only on the side of the latter). On the contrary, information seems to be much more "plebeian": only a particular scientific notion even technical and relevant to human artefacts, even having been invented only in the middle of the last century.

Once information has turned out to be able to unify "Body" and "Mind" and analogically to the same function of "Time", it advances towards the status of philosophical concept and

${ }^{4}$ Zermelo $(1904 ; 1908)$ inferred the well-ordering theorem from the axiom of choice being ostensibly more obvious than that theorem. The axiom was introduced by Zemelo especially for the proof of the theorem. However Whitehead and Russel in Principia mathematica demonstrated very soon (the very beginning of the third volume of Principia mathematica, even before the first enumerated statement in the volume: the proof is only a few strokes. and extremely elementary) that the well-ordering "theorem" implies the axiom of choice. 
even category. Thus, information is introduced as the counterpart of "time" as "Being", to "Time". However, the concept of information even outruns that of time as more general due to informational, but atemporal phenomena. "Entity" as a necessary projection of "Being" on "Time" can be generalized further, to those entities admissible to be out of time (or out of "Time").

All operators in the separable complex Hilbert space are its mappings onto itself. One can consider the definition area of any of them, which is always the separable complex Hilbert space (or respectively, the qubit Hilbert space), as the free variable of quantum information possessing any wave function (eventually represented as a series of qubits) as a certain value of itself. Then, any operator in that Hilbert space including the Hermitian ones (each of which is relevant to a certain physical quantity in quantum mechanics) would be a corresponding processing of quantum information.

If one consider the " $Z_{2}$ " Hilbert space (where " $Z_{2}$ " is a field in a rigorous mathematical meaning of "filed" and consisting of two elements) rather than the separable complex Hilbert space, any operator in it would be a real function. Then, the field of real numbers would be the analogical free variable of classical information. Consequently, quantum information can be granted to be "meta-information" or the "information of information" as far as its values are functions (that is: a certain operator of classical information).

Any functional ("functional" is used as a noun and mathematical notion) meant in quantum mechanics (e.g. as a certain physical quantity) is a mapping of the free variable of quantum information into the free variable of classical information. According to a possible definition of "dual space", it can be considered as the functional space toward the free variable of quantum information. The free variable of quantum information is just the space itself, to which the functional space can be accepted as dual.

The one Hilbert space consisting of all possible wave functions, interpretable as all possible quantum coherent states, contains those of the studied or investigated entity at issue. The coherent state of that entity can be granted to represent it "by itself" or "before measurement". On the contrary, its dual space contains all relevant mappings of its wave function into all possible results "after measurement". According to the uncertainty principle (interpreted widely), any wave function of the dual (functional) space contains only a single value relevant to the measured quantum state and its wave function.

A functional defined mathematically, the argument of which is the value of quantum information at issue (this means: in the initial space of states "by themselves"), possesses a certain value of classical information as a measured result referring to the state. The set of all measured results (i.e. each of them corresponding to a different functional in general) being a set of values of classical information with an attached probability for just it to be measured constitutes the initial and investigated wave function as the characteristic function of the probability density distribution corresponding to the set of measured values accompanied by the probabilities to be measured. 


\section{THE JOINT RELATVITIVITY OF THE FINITE AND INFINITE, EXTERNAL}

AND INTERNAL: THE RELATIVITY OF CLASSICAL AND QUANTUM INFORMATION

The main thesis of the paper icabout the relativity of classical and quantum information rooted just in the joint relativity of the finite and infinite, external and internal, in turn originating from the totality as it is postulated by scientific transcendentalism formally in the previous section.

The term "relativity" refers to the Einstein conception of relativity is not chosen occasionally. It is used to direct a further generalization of the same idea including a wider invariance also as to discrete reference frames relevant to quantum mechanics (Penchev 2021 June 8).

The general principle of relativity as it was formulated by Einstein means two reference frames which are two points in a smooth manifold: there exists always a single real smooth trajectory of mechanical motion able to connect the two points. That principle is called "general" because it admits for the two reference frames at issue to be accelerated arbitrarily to each other as well at to any intermediate reference frame associable with the smooth trajectory of motion between them unlike the "special principle of relativity" postulating the invariance of natural laws only to all inertial frames being non-accelerated mutually. Whether accelerated or inertial, all reference frames in both special and general relativity are not discrete: they are continuous necessarily, belonging to a smooth manifold. So, the conceptual correspondence of general relativity and quantum mechanics as a necessary condition for any theory of quantum gravity therefore unifying them implies a further generalization of the Einstein idea of relativity, which can be called the "still more general relativity", or the principle of quantum relativity:

It suggests the new concept of "discrete reference frame" meaning an ambiguous and even any arbitrarily unambiguous correspondence of two discrete reference frames linkable to each other only by an infinite set of virtual Feynman trajectories but not by a single one as all classical physics, special and general theory of relativity are restricted.

Information and mechanical motion (as well as any physical change) can seem quite different and unrelated to somebody, even to anybody who does not accept that principle of quantum relativity or does doubt in its reasonableness. On the contrary, granting the principle of quantum relativity implies the identification of information (especially quantum) and motion being obvious after the exhaustive interpretation of quantum mechanics as the theory of quantum information starting from the end of the last century.

Once the principle of quantum relativity and the concept of discrete reference frame have been involved, the joint relativity of the finite and infinite, external and internal as well as the relativity of quantum and classical information (therefore implying the generalized concept of information regardless of whether quantum or classical, i.e "information at all") can be inferred from that principle.

Joint relativity means that if the finite or the infinite are mutually transformed, and the external and internal are also mutually transformed (i.e. correlatively to the former transformation), the composition of both transformations (being commutative) is an identity. 
For example, if there exists a finite and external description, it is to be equivalent to a certain, infinite and internal description after the double exchange of finite and infinite, and external and internal.

However, that joint relativity is projected on the "screen of time" in default as far as time is granted to be a universal condition for any entity or about the being at all as well as for any physical entity or process in particular. Using the visualization of joint relativity by a bit of information, one can transcend the "temporal screen" as follows:

A bit transformed by joint relativity would exchange simultaneously: (1) its two alternatives of choice between themselves (for example, as " 0 " is transformed into " 1 ", and " 1 " into " 0 " in a tape cell of Turing machine or by the logical operation of negation); (2) the inseparable state before choice and the separable state after choice and consisting of two alternatives (for example, an empty cell of Turing machine and the same sell after recording either "1" or "0"). Speaking loosely, the bit would change its direction. If it is projected on the temporal screen definitively, this implies a more complicated, ternary joint relativity including additionally the direction from finiteness to infinity (resp. vice versa) as third element together with the first two ones: the finite and infinite, the external and internal:

The mutual interpretation of both alternatives of a bit of information as the external and internal in relation to each other seems to be obvious, an "axiom". The interpretation of the state (before choice as infinite, and that after choice as finite) is not so obvious: it needs to be postulated philosophically or justified mathematically by the equivalence of the axiom of choice and the well-ordering "theorem" in set theory.

As to the third element of the triple joint relativity, the "direction of a bit" (also interpreted as in the previous paragraph) can be understood as temporality rather metaphorically in an ontological sense (even in a theological sense valid in Christianity) than in a literal sense referring to the physical quantity of time. Nonetheless, this can be conjectured being inferred in a more rigorous way in another paper (Penchev 2021 June 8).

If one grants, that triple joint relativity (respectively the invariance to the joint exchange of its tree binary oppositions), both kinds of information, classical and quantum (as far as they are relevant to the one opposition, namely that of finiteness and infinity) can be considered to be also "relative" in turn therefore suggesting their shared species of information at all distinguishable as whether classical or quantum only under the condition of exchanging only one of the rest two oppositions: either the external and internal or the direction of time. The simultaneous exchange of both impies just the indistinguishability of classical and quantum information.

For example and on the contrary, the postulation of the temporal screen (e.g. as physics does by energy conservation equivalent to the "screen of time") guarantees for classical and quantum information to be clearly and absolutely distinguishable. 
V TRANSCENDENTAL TIME: AN EPOCHÉ TO THE DIRECTION OF TIME ARROW, BUT NOT TO THE TIME ARROW ITSELF

Husserl introduced his innovative concept of "epoché" as an attitude to reality, called to abandon the question whether whatever entity is real or not. Thus, it is to direct the entity by itself, "thing in itself", Husserl's "Zur Sache selbst!" or Kant's "Ding an sich" therefore creating a generalized, modified, or equivalent version of transcendentalism as what his doctrine of phenomenology can be interpreted and will be interpreted further in this paper:

"Epoché" corresponds to an operation, "phenomenological reduction" resulting in the "phenomenon" of the entity at issue and meaning the invariant of that entity regardless of whether it is real or not. So, the concept of "epoché" involves s kind of philosophical relativity relevant, first of all, to modern Western philosophy originating from Descart's dualism: indeed, epoché is defined so that it is able to "bracket" the question whether the entity at issue is "bodily" or "mental".

"Epoché" can serve especially effective as a bridge between the classical philosophical transcendentalism (even sacralized and thus tabooed by history of philosophy) and its interpretation and use here as a falsifiable conception, "scientific transcendentalism" and thus, to an ontology of information also elaborated here.

In fact, the concept of "epoché" contains a very fruitful implicit uncertainty or ambiguity also interpretable as a second relativity and therefore able to constitute a joint relativity being unifiable with the former (about reality and unreality) and thus quite relevant to the joint relativity discussed in the present paragraph and sharing the same formal structure as a bit of information.

This binary ambiguity, opposition, or second relativity, refers to the unity of the alternatives (reality and unreality): either as the (logical) disjunction of them as the pair of the two possible states of a bit of information (e.g. similar to the pair of " 0 " and " 1 ") or as their (logical) conjunction, the shared invariance of "phenomenon" being zero as to the individualized, disjunctively separated alternatives meant in the former option for the binary ambiguity to be understood. On the contrary, the latter option suggests that the (logical) conjunction of the alternatives is not zero, and even coinciding with each of them therefore implying their identification as the extreme opposite to that of their ultimate disjunctive separation meant by the former option.

Quantum mechanics reformulated thoroughly as a theory of quantum information suggests furthermore a terminological visualisation of the gradual transition from the former option to the latter as the decoherence of any coherent state: an entangled qubit (on the one extreme it is maximally entangled) passes smoothly into the disjunctive separation of two qubits (on that opposite extreme, the entanglement is zero).

Utilizing the picture in terms of qubits and quantum information in the last paragraph one can describe the same process of gradual decoherence by the terms of the "classical" quantum mechanics and its mathematical formalism of the separable complex Hilbert space.

${ }^{5}$ Even Husserl's idea or approach about "Philosophie als strenge Wissenschaft", once his phenomenology be understood as a kind of transcendentalism, can and even should be interpreted as generating "scientific transcendentalism" using the term coined here. 
The process of decoherence includes two hierarchical stages: (1) decreasing the number of the "axes" of the separable complex Hilbert space sharing any nonzero entanglement (respectively, increasing the number of orthogonal "axes"); (2) decreasing the degree of entanglement of each one "axis", in which entanglement is nonzero, until it reaches zero (respectively, increasing the mutual rotation of each pair of two "namesake", or "numbersake", axes until they turn out to be orthogonal to each other).

One can state that the physical quantity of time with all properties inherited from the namesake quantity in classical physics exists only at the one extreme, that of two disjunctively and absolutely separated alternatives. Consequently, just that extreme is to be identified as the temporal screen granted by classical physics to be a necessary condition for any physical process or entity. For example, gravity can be interpreted as the projection of the entire process of decoherence running gradually and smoothly all intermediate states of decreasing entanglement on the temporal screen of only the one extreme of complete decoherence $^{6}$.

Then the process of decoherence is what creates time in such a way that time appears in each moment of time again and again ${ }^{7}$. The decoherence itself is out of time, but nonetheless it is a physical process therefore belonging to a new and more general nontemporal physics, to which all the standard temporal physics is not more than a particular case, extreme and borderline, at that.

If the temporal screen is granted as a necessary condition of physics, the process of decoherence and occurrence of each successive moment of time is not accessible directly and explicitly since decoherence is a meta-process in relation to time and generating it. Nonetheless, it will be observable by its consequences, to which its projection as a hypothetical beginning of the universe, namely the notorious "Big Bang":

It is a real event in the framework of the standard temporal physics (what all physics until now is). On the contrary, it can not be a real event, but only an aggregate total projection of all the processes of decoherence having taken place in the universe until now into a single and unique moment of time (its ostensible beginning, properly).

So, the Big Bang is rather a myth called by the limitation of temporal physics than a real fact once the new generalizing non-temporal physics is adopted. Moreover, it is the third "dark" phenomenon (along with "dark matter" and "dark energy") and can be renamed the "dark beginning" of the universe since all current violations of energy (mass) conservation are removed from physics once the Big Bang has happened somehow and concentrated into

\footnotetext{
${ }^{6}$ Meaning general relativity as the relevant theory of gravity, the same "screen of time" is Einstein's (1918) "Mach's principle" stating that mass and energy are the only sources of gravity. Then, energy (mass) conservation and Emmy Noether's (1918) "first theorem" implies the "temporal screen", only on which any gravitational process can become visible. Respectively, any other source of gravity, different from mass and energy (if any) would be projected on that screen (since general relativity is granted as valid) as "dark matter" and "dark energy", experimentally very well corroborated nowadays. Another paper of mine (Penchev 2020 October 20) conjectures entanglement as that additional source of gravity.

${ }^{7}$ That worldview corresponds to Descartes's idea (Descartes 1648; Mamardashvili 1981; Gorham 2007 ; 2008) that God creates the world permanently, in each moment of time rather than once, at the unique act of the Creation.
} 
it. Then, the question and problem of how it might have occurred after it is a gigantic violation of energy conservation is forbidden.

However, all the three most fundamental "dark phenomena" furthermore refer to about $95-96 \%$ of the universe and even to its existence, and appear only on the screen of time, on which they are visible only as "dark shadows". The reason for them to seem only as "dark shadows" is rooted in our cognition, more specially, in the temporal screen, which physics and all experimental science unfurls as a necessary condition for any knowledge claiming to be scientific, rather than in their nature by themselves.

Well, what about their nature by themselves?

Their substance is information rather than matter and energy: information is the most fundamental substance therefore able to comprise both physics until now being temporal and all "dark phenomena" being non-temporal, and to investigate their structure, which is informational rather than material (i.e. physically energetic).

The present section, however, is directed only to the ontological projection of that generalization relevant to philosophy rather than to physics, at that utilizing Husserl's phenomenology and, first of all, his concept of "epoché" as possessing the formal structure of a bit of information (demonstrated above in the present section) and thus presumably suitable for establishing the corresponding ontology of information by those "units of epoché" turning out to be "bits of information", at least in the sense of mathematical isomorphism.

Accordingly, one need define the philosophical category of Time ${ }^{9}$ corresponding to both Husserl's "epoché" though interpreted informationally as here and the joint "triple relativity" also described above in the present section. Then, Time is to be the complement (in a wider sense though similar to the meaning of the term in set theory) of that epoché explicated informationally to the triple relativity.

In other words, which would more relevant to the contemporary physics and its commitment to the temporal screen, Time refers to the way for the temporal screen to be reformulated though only philosophically so that it to be consistent to the triple relativity (in turn linked to information at all, i.e. regardless of being whether classical or quantum).

Time (by a capital letter) can be understood (especially in the present context considering phenomenology as a bridge between transcendentalism and science as empirical and experimental as postulative and deductive) as transcendental time and consistent with "information at all". It is related directly to the totality able to unify the time of its internality with that of its externality though directed oppositely to each other in virtue of the uniforming capability of the totality originating from its definition for scientific transcendentalism.

\footnotetext{
${ }^{8}$ The allusion is to the well-known metaphor of the "cave, on the wall of which chained people observe only shadows" and suggested by Plato.

${ }^{9}$ The capital letter of "Time" is to distinguish it from the physical concept or quantity of "time", however, only in the way to be its metaphysical counterpart therefore postulated to be unfalsifiable though coinciding with its contemporary "twin": scientific, falsifiable and refutable by experience or experiments.
} 
That transcendental time means pure well-ordering therefore needing a new, "temporal epoché" on relation of both possible time arrows (i.e. "forwards" versus "backwards"), or respectively: the two opposite and idempotent well-orderings, which are possible always once any of both is given or postulated. So, transcendental time and temporal epoche are to be correlative to each other.

Transcendental time is especially relevant and necessary to the philosophical transition from temporal physics (as it is until now) to a future and generalizing non-temporal physics therefore tracing the pathway of a proper scientific, physical and mathematical transition. The reason is that transcendental time allows for the temporal screen of contemporary physics to be reformulated and thus generalized in a way valid for non-temporal physics thus delivering rigorous formal rules for translating between the temporal and non-temporal languages of physics.

The essence of those rules consists in the identification of both possible and opposite directions of ordering or time arrow with a single one being "phenomenal" in a Husserlian sense after the newly temporal epoché. Thus, the rules utilized again the formal structure of a bit of information already introduced in advance in virtue of the informational interpretation of epoché as a little above.

An essential additional question relates to what the theory of quantum information complements to quantum mechanics as to the temporal screen reinterpreted by transcendental time. Indeed, quantum mechanics has involved implicitly the temporal screen (respectively, "conserving energy conservation") of Txe contemporary physics even being recorded in its "Tablets of Stone" (what the separable complex Hilbert space is for it) as "God's Command" of untaritity ${ }^{10}$.

Then, what is sufficient for the theory of quantum information to complement is only articulating explicitly the structure of bit of information implicitly involved in the structure of the separable complex Hilbert space as the pair of two complimentary, but namesake ("numbersake" or "ordinal-numbersake") axes, each of which belonging to the one dual space $^{11}$. In fact, that articulation does not add anything new to quantum mechanics, but it is very suitable to the thinking of human beings, furthermore supplying a more convenient technical apparatus by the concept of quantum information and its quantity measurable in units of qubits and being the substrate of entanglement.

\section{TRANSCENDENTAL TIME AND ITS CORRELATE OF INFORMATION: PHYSICAL ACTION}

A conclusion from the previous section links as correlates two pairs: (1) the usual physical quantity of time and energy obeying the law of conservation both being relevant to the temporal screen of physics until now; (2) a new understanding of the physical quantity of

\footnotetext{
${ }^{10}$ Intarity and the corresponding "conservation of energy conservation" in quantum mechanics are discussed in detail in a previous paper (Penchev 2020 October 5).

${ }^{11}$ Still the "triple article" of Einstein, Podolsky, and Rosen (1935) demonstrates that the separable complex Hilbert space of quantum mechanics (if one dare admit for it to be complete) implies "spooky actions at a distance" (by Einstein's pictorial metaphor), i.e. physical actions accomplished non-temporally and therefore out of the scope of temporal physics definitively.
} 
time managed by the idea of transcendental time and information at all, that is information whether quantum or classical. The latter refers to a new, suggestable, but nonexistent yet area of atemporal physics including current physics as the particular case as occurring or projectable on the temporal screen not being a necessary condition for physics already.

The present section is concentrated on the description of the new understanding of "physical time" corresponding to the rather philosophical "transcendental time" as well as to the presumptive generalization of energy conservativation to information conservation or to quantum-information conservation and its identification with physical action.

Comparing the usual understanding of "physical time" (as a concept as a quantity) and what would be relevant to its philosophical counterpart of "transcendental time", the difference seems to consists only in the independent complement of the formal structure of a bit of information, at that comprising the usual physical time of the hierarchically next level, i.e. the metalevel:

Time is doubled by its complementary counterpart running "backwards" and both constitute the two possible disjunctive alternatives of a bit of information. Their inseparable state (e.g. as an "empty" tape cell of Turing machine) preceding the choice of any of both alternatives corresponds to reversible time (unlike any of both opposite irreversible times) and thus to a coherent quantum state.

Adding the metalevel of time modifies necessarily the formal structure of a bit of information transforming it into the qubit Hilbert space and thus, into the usual separable complex Hilbert space of quantum mechanics. Here is why and how:

Any moment of time, which can be thought of as "global time" for the next consideration and distinction, is to contain the complete time within itself as "local time". This follows from the integration of the formal structure of a bit of information namely and just in the metalevel, i.e. as a "meta-time" therefore needing a second understanding of global time as a complete process ${ }^{12}$, at that embedded cyclically into the lower hierarchical level, i.e. that of proper time as local time.

However, an infinite set of different infinite ordinals corresponds to the complete time, and each of those ordinals can be embedded as a "local time" into a certain moment of "global time". Since quantum information can be considered equivalently as the information of infinite series or sets (Penchev 2020 July 10), one can prove ${ }^{13}$ that exist a bijection of all qubits and all infinite ordinals (which can be restricted only to countable physical ordinal numbers as to the physical theory of quantum information). So, a qubit (different in general) would represent the local time within any moment of global time.

The mathematical structure corresponding to the physical counterpart of "transcendental time" turns out to be the qubit Hilbert space and thus the separable complex Hilbert space of quantum mechanics. That interpretation suggests another philosophical and scientific, physical and mathematical perspective to the extraordinary solution of quantum mechanics:

\footnotetext{
${ }^{12}$ The understanding of time as a complete process corresponds exactly and even formally to the concept of infinite set in set theory as actual completeness.

${ }^{13}$ The rigorous mathematical proof of the statement is to be demonstrated in a future work.
} 
The "scientific common sense" grants that quantum mechanics involves a special mathematical formalism relevant to its special subject as all physical theories and hypotheses have done and continue to do. However, if one as here interprets the formalism of quantum mechanics as utilizing a mathematical structure suitable to a new generalizing understanding of physical time, a quite different and extraordinary viewpoint is opened and it should be compared in significance with the infinitesimal revolution of Newton and Leibniz to physical cognition.

Infinitesimal calculus is an instrument applied exceptionally successfully initially in Newton's mechanics and theory of gravity therefore creating the paradigm of physics in the next centuries. As its ontological justification, one can suggest the relevance of infinitesimal calculus to represent mathematically any process of time, what any physical ones have seemed to be since Newton's age until the experimental confirmation of entanglement. So, created both theory of gravity and a paradigme of physics relevant to postulating the temporal screen as a necessary condition. Common sense means quantum mechanics to be still one theory in the fundamental pathway of Newton postulating the temporal screen and infinitesimal calculus as obligatory and necessary to physical cognition regardless of the subject of the theory or hypothesis at issue.

Quantum mechanics appeared immediately in two forms in the mid twenties of the last century: Heisenberg's matrix mechanics and Schrödinger's ondulatory mechanics. The latter seemed to be in the Newton paradigm applying the infinitesimal calculus to a new subject, wave function, presumably relevant to quantum mechanics. On the contrary, Heisenberg's matrix mechanics was fundamentally discrete being relevant to quantumness, but not to the universal continuous time embedded in the application of infinitesimal calculus to physics.

Quantum mechanics unified both involving the separable complex Hilbert space and its property of unitarity quite pertinent to the "conservation of energy conservation" in its scope thus keeping the Newtonian temporal screen to quantum mechanics as well. However, Einstein, Podolsky, and Rosen demonstrated that the separable complex Hilbert space implies the "spooky action at a distance" of entanglement. Indeed, the three authors interpreted it not as the prediction of a real physical phenomenon, but as reductio ad absurdum in relation to the completeness of quantum mechanics due to the obvious nonsense of entanglement as a physical phenomenon (according to them).

In fact, quantum mechanics turns out not to be incomplete, the phenomena of entanglement are very well confirmed experimentally nowadays, but nonetheless the Newtonian temporal screen was conserved as to quantum mechanics and developed in the Standard model. Only the discovery of dark matter and dark energy paid our attention to the inconsistency of quantum mechanics and the temporal screen.

Meaning that background, one can admit that quantum mechanics like Newton's universal gravitation suggests both physical theory and paradigm therefore generalizing the apparatus to infinitesimal calculus to operators in the separable complex Hilbert space due to generalizing the Newtonian temporal screen to atemporal physical phenomena what all those of entanglement are. The meaning of that Gestalt change to quantum mechanics is considerable and even revolutionary: many other theories and hypotheses both within and 
out of the scope physics can follow the new, atemporal paradigm suggested by quantum mechanics and its doubtless success ${ }^{14}$.

If one process, whether physical or not, involves a wave function for its description, it happens within the temporal screen only partly. Another part of it takes place out of that screen though it possesses or can possess some "dark projections" on it similar to the way entanglement is projected as "dark matter" and "dark energy" in the paradigmal theory of quantum information.

Furthermore, applying the apparatus of the separable complex Hilbert to any phenomena and following the cognitive pattern of quantum mechanics, one can investigate how the time specific to them and the corresponding orderings, i.e. information and thus selforganiztion appears by themselves (i.e. as causa sui) even in the simplest physical systems (which are not mental or social; not biological or chemical where organization caused externally or internally is researched).

On the contrary, the usual and commonly accepted approach for all natural sciences to be postulated time, even single and universal, closes any access to how time can appear and may appear within the studied system at issue. When that approach is forced to investigate the natural phenomenon of how the temporal screen and time itself have come into being, myths as the Big Bang are unavoidable in order to be concentrated what is out of time only within that unique moment of time, in which all arises out of nowhere, but after which time does not arise any more and its screen is postulated to exist forever and as a necessary condition for any natural process or phenomenon.

That more general approach advocated here replaces time with transcendental time or scientifically, physically and mathematically, infinitesimal calculus and differential equations with the qubit Hilbert space of quantum information and corresponding operators defined on it. If the quantity of discreteness (e.g. such as that is forced by the Planck constant) tends to zero, the more complicated (latter) model passes gradually and smoothly in the former one.

However, quantum mechanics is not still one physical theory following the thalweg and track established by Newton by his infinitesimal calculus applied in relation to universal gravitation. On the contrary, quantum mechanics was forced to create a new, "quantum calculus", which it applied to its subject therefore suggesting a new paradigm of physical and scientific cognition.

Properly, the physical correlate of transcendental time is to be quantum information and even, the substance of quantum information thus emphasizing that quantum information conserves similarly to energy and generalizing the conservation of the latter in natural sciences. The usual time and its corresponding physical quantity progresses steadily only and being featured definitively by time arrow therefore suggesting a corresponding Lie group and the conserving quantity of energy as in the first Noether (1918) theorem.

However, the physical correlate of transcendental time is deprived of the unambiguity of time arrow in virtue of being a generalization. Nonetheless and just due to the generalization, it can keep the property of universality (inherited from the understanding of the temporal

\footnotetext{
${ }^{14}$ A review of possible applications of the new paradigm and not claiming exhaustivity contains
} another paper (Penchev 2021 February 25). 
screen as a necessary condition) as the corresponding conservation of the physical correlate of transcendental time, i.e. quantum information. The latter is a quantity dimensionless physically and can be interpreted as a generalized time derivative of time making sense and rigorous mathematical meaning even after any discrete temporal leap where the standard defined time derivative of time ${ }^{15}$ would be uncertain or assume a value of infinity. Then, the conservation of quantum information can be interpreted as expressing the obvious conservation of the distance of a series of quantum leaps if they constitute a single jump in the final analysis.

Being physically dimensionless, the correlate of quantum information in the first Noether (1918) theorem is to be the physical action itself, and the conservation of the former suggests a Lie group of the accumulated physical action, however that accumulation cannot not happen during time since time and its "screen" is not more a necessary condition in physics. It can be considered rather as a mathematical corollary from quantum information conservation after it has been adopted as a universal physical law. Furthermore, physical actions (e.g. those featuring all phenomena of entanglement or Einstein's "spooky actions at a distance") taking place out of time are to be admitted as a rule rather than as exceptions or rejected at all.

Since the unit of quantum information is a qubit, and the natural unit of physical action is the Planck constant, the eventual correlation of quantum information and action in virtue of the first Noether (1918) theorem implies the correlation of their units, e.g. proportionality, and its coefficient to be an arithmetical unit, " 1 " is the simplest case, at that no objections against this are known.

Properly, the physical action corresponding to information (at all) unifying quantum information equivalent to nonzero physical action and classical information not possessing physical action might generate some inconsistency at first glance:

That unification relies on removing the screen of time as a necessary condition, and energy (as well as mass) can be defined and observed only on its screen. The conservation of quantum information suggests that it can be partly or even thoroughly transformed into classical information in virtue of their identification as "information at all". This implies that not only energy, but also action can change itself in any physical system by itself not needing any cause different from abandoning the temporal screen.

Another explanation is not less possible: universal entanglement excludes for any physical system to be closed and conservative for that property is definiable only within or on the temporal screen. For example, a "causal" reason out of the temporal screen can be any instant interaction, among which can be enumerated effectively those with a future state or

\footnotetext{
${ }^{15}$ The standard defined time derivative of time would be a finite constant, conventionally a unit, as to the Newtonian time and a function of the variable of the three-dimensional space velocity as to special and general relativity. There exists a mapping, even a bijection under a few additional conditions, between the time derivative of time in relativity and it is expressed by the tool of quantum information. Furthermore, the constant time derivative of Newtonian time can be generalized to "transfinite constants" (representing transfinite ordinal numbers) so that any transfinite constant is a certain wave function and thus a certain value of quantum information.
} 
with an arbitrarily remote past state: both are to be situated out of the light cone of special relativity ${ }^{16}$.

One can admit even that mass at rest can be defined or explained by entanglement and as the total interaction originating from the area out of the light screen; this means: as the equivalent of the entire dark mass due to entanglement concentrated within the entity possessing that mass at rest in question. Then, the problem about the difference of the visible mass at rest and dark mass would appear for both are due to entanglement. A possible explanation can be the following:

There are two different reference frames: that of the studied entity and the observer's one, and as if two different light cones are associated with each of those reference frames. Nonetheless, the two light cones can be identified as the same just in virtue of the theory of relativity (whether special or general). Physical transcendentalism is able to elucidate that the alleged contradiction is only seeming: in fact, relativity has established physical transcendentalism implicitly, by means of distinguishing disjunctively either quantities invariant to Lorentz transformation and introduced only by special relativity or non-invariant to it and traditional for physics, such as time, space coordinates, mass, etc.

The former ones do not undergo any change after the transition between the two reference frames and are to be related to the same cone of light, though shared by two reference frames. On the contrary, the latter ones are subject to change being Lorentz non-invariant quantities. This is the description of special relativity modified by general relativity not in essence.

What would be to follow is the new, generalizing description of quantum information introducing the quite different concept of "discrete (or external) reference frame". It grants that the reference frames can interact instantly (that is out of the temporal screen), super-luminally rather than only sub-luminally (that is within the temporal screen and the scope of Einstein's relativity). Then, that part of entanglement due to the interaction within the same and shared light cone could be visible and measurable as the quantity of mass at rest. The other part of entanglement due to interaction out of that light cone could condition dark mass and dark energy properly.

In other words or as an example, mass at rest ${ }^{17}$ is due to the instant entanglement with the visible universe ${ }^{18}$ and dark mass and dark energy would be due to the entanglement with the

\footnotetext{
${ }^{16}$ The superluminal "half", i.e out of the light cone (i.e. the real domain of Minkowski space) can be mapped as a homeomorphism (consequently, bijectively) onto the subluminal "half" within the light cone of pseudo-Riemannian space rather than only within the light cone of Minkowski space. Furthemore, the superluminal "half" of pseudo-Riemannian space doubles its subluminal "half" and can be interpreted as the tensor corresponding to the energy-momentum of gravitational field and therefore, causing the deformation of the homeomorphism at issue. The relevant physical interpretation linked to entanglement is discussed in: Penchev 2013.

${ }^{17}$ Mass at rest corresponds only to the space-time component (Ricci tensor) of entanglement, and gravitational field, to its energy-momentum component (the corresponding tensor in the Einstein field equation). That conclusion can be inferred following the approach considered in detail in another paper (Penchev 2020 October 20).

${ }^{18}$ Mass at rest remains constant though the volume of the visible universe, and thus the interaction of entanglement increases, since the standard unit of mass at rest increases just in the same way and due to the same reason as the measured or investigated mass at rest.
} 
invisible universe. A corollary from the conjecture is that mass at rest, gravitational field, dark matter and dark energy share the same origin: from entanglement.

\section{CONCLUSIONS}

Two preliminary notices are necessary to prevent eventual objections to the conclusions. The one of them refers to the "eclectic genre" of the paper, and the other, to the necessary generalization of causality in relation to all phenomena of entanglement or involving quantum information: however that generalization is neither "mystical", nor "anti-scientific", nor it "destroys causality", but only defines it in a new way able to include the current understanding of causality as necessarily temporal as a particular case.

Paper involves the seemingly "too speculative" premise of the totality as a postulate implying direct corollaries in the deductive and axiomatic foundations of mathematics as well as in the experimentally testable foundations of quantum mechanics, and thus of physics. So, the demarcation line preventing the direct conflict of philosophy and science turns out to be not more than one prejudice. "Crossing that red line" can be justified so:

(1) Science (even not only physics and mathematics meant here expressively) needs the postulate of the totality once its development forces it to go out of the specific "cognitive niche" inhabited by humankind and where human mind has arisen in accordance with immediate experience initially, and then, with very special, sophisticated, and expensive experiments (as in physics today). In fact, that permanently crossing the red line of the current experience has featured modern science since its occurrence in the mid of the last millennium calling always attacks and resistance against it (for example by religion, but not only).

However, the now undertaken step is much more radical in a sense: it crosses as a prejudice one of the most fundamental principles constituting empirical science: the condition sine qua non of time. The postulate of the totality is what allows for cognition to be directed and controlled once the thread of time has had to be abandoned. This is not too dangerous since humankind has developed mathematics absolutely successfully for a few millennia out of the temporal screen of empirical experience.

In fact, the "red line" distinguishing physics and mathematics, or reality and model, is crossed as a visible result following the postulate of the totality. Properly, still the theorems of the absence of hidden variables in quantum mechanics (Neuman 1932; Kochen, Specker 1968) melted irrevocably that boundary as a necessary logical corollary once the separable complex Hilbert space (being inherently unitary) has been established as the mathematical foundation of quantum mechanics.

(2) That same unitarity implies non-temporal phenomena to be physical (a ridiculous state of affairs as to classical physics and science and painted wonderfully by Einstein's metaphor of "spooky action at a distance"). Indeed, the unification of Heisenenberg's discrete matrix mechanics and Schrödinger's smooth ondulatory mechanics in the contemporary quantum mechanics is possible only under the condition of unitarity and means any reversible (and thus non-temporal) coherent state before measurement to be mapped as an irreversible (and thus temporal) observed experimentally state therefore necessarily reducing (or "losing") just the half of variables required to be described exhaustively the former state. 
After the cognition of quantum mechanics has been commonly accepted to be adequate, the former state of $2 \mathrm{~N}$ variables and the latter state of $\mathrm{N}$ variables are linked bijectively therefore involving infinity ${ }^{19}$ (one of the most fundamental mathematical concepts) into experimental physical science (what quantum mechanics is) and factually "crossing the red line" of physics and mathematics as an embodiment of that between philosophy and science and neant in the notice 1 above.

Thus, the mapping of $2 \mathrm{~N}$ variables into $\mathrm{N}$ variables turns out to be a bijection if the set of $\mathrm{N}$ variables would be infinite. However, it is certainly finite in classical mechanics as far as those $\mathrm{N}$ variables refer to some system consisting of a finite set of $\mathrm{M}$ subsystems (called "bodies" in tradidition). So, one needs an explanation or respectively, a constructive mechanism of how the "finite" classical mechanics to be reconciled and consistent with the "infinite" classical mechanics. The same idea considered properly mathematically means the logically correct way for any finite set to be equivalent to a certain infinite one and thus, vice versa as well. The only well-known tool in the foundations of mathematics is the so-called paradox of Skolem ${ }^{20}$ or the set-theoretically defined finiteness (or Dedekind's finiteness).

As to quantum mechanics, the nonclassical, "2:1" bijection of finite sets is involved implicitly by the unitarity of the separable complex Hilbert space (which is the main reason for it to be utilized as its fundamental mathematical formalism) and proved explicitly to be a bijection in virtue of the theorems of the absence of hidden variables in quantum mechanics ${ }^{21}$ (Neumann 1932; Kochen, Specker 1967). That solution can be demonstrated in its essence elementary:

The bijection of the kind " $2: 1$ " is to be simultaneously a homeomorphism (since any bijection is a homeomorphism) and discrete (or, "quantum"), "jump-like" since any transition of two elements to a single one is a leap. Those two properties seem to be contradictory thus implying the corresponding set satisfying both as its characteristic property to be empty.

${ }_{19}$ More precisely, the property of an infinite set to be bijectively mappable into its true subset, particularly, into that consisting of the exact half of elements of the former one.

${ }^{20}$ I mean the famous presentation of Skolem (1922, published in 1923) where he formulated his notorious "relativity of the concept of set" (italic is mine) inferring that any cardinal number of an infinite set (and he expressly declared: "even finite" in the same paper, though the finiteness at issue can be only the set-theoretical, "Dedekind finiteness") is relative as a direct corollary from the theorem called today the "Löwenheim - Skolem" one in turn valid in virtue of the axiom of choice in set theory. That relativity means that any cardinal number can be considered to be of an arbitrary power even a finite one once the axiom of choice has been granted. The present paper attempts to generalize Skolem's relativity of "set" further, from the foundations of mathematics to philosophy since other papers (Penchev 2020 July 1; Penchev 2011) have described its application in quantum mechanics isomorphically.

${ }^{21}$ Indeed, if "no hidden variables" is the case, the "second half" of any " $2: 1$ " mapping turns out to be redundant and thus the mapping, a bijection. The interpretation of that conclusion to the fundamental epistemological relation in science, namely "reality - model" (respectively, to Descartes "mind-body" problem, or that of "object - subject" in German classical philosophy) is especially important because it implies that reality and model can be identified under the condition for the model to be "infinite" (as in quantum mechanics), and the corresponding problems constituting the episteme of modernity to be resolved rigorously and mathematically (a message proclaimed still by Husserl). 
However, quantum mechanics displays that the contradiction refers only to the prejudice of classical physics and science and involves the simplest (conjecturally) possible model of the separable complex Hilbert space able to realize the $2: 1$ bijection at issue consistently being forced to that radical step by the necessity for unifying the homeomorphisms of the apparatus (described by classical physics) and the newly, quantum and leaplike changes of any quantum entity.

One can emphasize that infinity is involved in quantum mechanics (though it studies systems consisting of a finite number of entities, just as classical mechanics does) in virtue of the jump-like reduction of $2 \mathrm{~N}$ to $\mathrm{N}$ variables (where $\mathrm{N}$ is a finite natural number). Classical mechanics conserves $2 \mathrm{~N}$ variables to a certain system both before measurement and after it therefore not needing that jump-like reduction involving implicitly infinity. On the contrary, infinity is crucially important for quantum mechanics to be able to remain an objective science.

One of the most painful transformations called by quantum mechanics refers to the "acausal revolution" even questioning whether it can be a science inherently causal unlike religion. Indeed, any single result of its measurement is fundamentally random: this can be tracked still to the axiom of choice, as above. Anyway, causality can be conserved to the probability density distribution of all possible measurements ${ }^{22}$ though neither to a single one nor to any finite set of them, what the real measurements are always.

That generalization of causality to probability density distribution can be developed further in the present context interpreting it as reverse causality by itself (i.e.: backwards in time), but pictorable as an unambiguously certain probability density distribution as we observe it (being the only way and necessary condition for our experiments engaged with the just one time arrow: that "forwards" of our experience).

Speaking loosely, the classical casualty of determinism is able to describe not more than the "half of reality": the other half remains fundamentally random in our experience, being due to reverse causality involved by quantum mechanics by its cognitive co-temporal image ("forwards" in time) of probability density distribution.

Human experience has arisen just as relevant to that "deterministic half of reality" as far as it can be influenced by human actions and thus directed in a way favorable for survival. The other, "fundamentally random half of reality" though not less essential for survival is abandoned by evolution and human experience originating from it in the final analysis being non-influenceable by any human actions. Anyway, humankind created religion existing in such great diversity and along with science today in an attempt to influence somehow the other, "divine half of reality" in a wishful direction like the former one accessible immediately by human experience.

Alas, though that "divine reality" seems to exist, even in a rigorous scientific and experimental meaning, it cannot be impacted by religious experience, rites or rituals of any kind existing anywhere all over the world: just as, speaking figuratively, gravitation cannot affected by any prayers even in a minimal degree. The "divine reality" obeys the same kind

\footnotetext{
${ }^{22}$ Max Born (1948) suggested the probabilistic interpretation of wave function was one from the first physicists showed that way out and generalization of casualty.
} 
of scientific and mathematical laws acting necessarily and independently of human emotions and spells, but only much more complicated than those ruling the "profane reality" of our empirical experience.

Humankind managed to reach to that hidden, known until now as "divine reality" by reliable scientific methods rather than in virtue of powerless religious worship only and hardly, hardly at and about the threshold of the third millennium by quantum mechanics and information and the shocking experimental discovery of "dark matter" and "dark energy": human cognition by science had outrun the natural evolution of human experience so much that it reached the sphere of religious experience: that of the fundamentally random events due to the reverse causality proclaimed by quantum information.

Just this is the main and most essential conclusion of the paper: the "divine" reality meant by religion can be defined scientifically and can be studied and investigated even experimentally by absolutely reliable scientific methods.

Science is able already to recognize the divine reality accessible until now only by the unsecure approach of religion of any kind. Nonetheless, this does not overcome the opposition of science and religion (maybe even, on the contrary) since religious worship of God is not less incombinable with scientific research and methods in relation to Divine Reality (according to religion unlike the ostensibly "divine" reality according to science) than to the profane reality of empirical experience meant by science until now.

The same step to the ostensibly divine reality seen properly philosophically consists in scientific transcendentalism formulated as in the present paper as in previous ones: it is the alleged "profane" counterpart of philosophical transcendentalism distinguishing from the latter by its unambiguous formulation therefore allowing for any refusal due to inconsistency or mismatch to experiments.

The more specific (or properly scientific) conclusions refer to the joint relativity of the finite and infinite, the external and internal, and quantum and classical information constituting together a more fundamental (even the most fundamental) invariance interpreting the conservation of quantum information from a methodological and philosophical viewpoint. The same relativity can be interpreted in turn still wider, namely as the relativity of the divine and profane, human being and God, science and religion, the fundamentally random and deterministic, the usual causality "forwards in time" and reverse causality "backwards in time" as the previous two notices in the section of conclusion sketched concisely. 


\section{References:}

Born, M. (1949) Natural philosophy of cause and chance being the Waynflete lectures delivered in the college of St. Mary Magdalene 1948. Oxford: Oxford University Press.

Descartes, R. (1641) Meditations on First Philosophy with Selections from the Objections and Replies (transl. M. Moriarty). "Oxford World's Classics". Oxford: University Press, 2008.

Einstein, A. (1918) "Prinzipielles zur allgemeinen Relativitätstheorie," Annalen der Physik 55 (4): 241 - 244.

Einstein, A.. B. Podolsky, and N. Rosen (1935) "Can Quantum-Mechanical Description of Physical Reality Be Considered Complete?” Physical Review 47 (10):

$777-780$.

Gorham, G. (2007) "Descartes on Time and Duration," Early Science and Medicine 12 (1): $28-54$.

Gorham, G. (2008) “Descartes on God's Relation to Time," Religious Studies 44 (4): 413 - 431.

Heidegger, M. (1927) Sein und Zeit. Halle an der Saale: M. Niemeyer.

Kolmogorov, A. N. (1965) "Three Approaches to the Quantitative Definition of Information," Problems of Information Transmission 1 (1): 1-7.

Kolmogorov, A. N. (1968) "Logical basis for information theory and probability theory," IEEE Transactions on Information Theory 14 (5): 662-664.

Kolmogorov, A. N. (1963) "On Tables of Random Numbers," Sankhyā: The Indian Journal of Statistics A 25 (4): 369-376.

Kolmogorov, A. N. (1998) "On Tables of Random Numbers," Theoretical Computer Science 207 (2): 387-395.

Mamardashvili, M. (1981) Cartesian Meditations (January 1981). (Editor: U. P. Senokosov). Moscow: Progress (in Russian: М. Мамардашвили. Картезианские размышления).

Martin-Löf, P. (1966). "The definition of random sequences," Information and Control 9 (6): 602-619.

Noether, E. (1918) "Invariante Variationsprobleme," Nachrichten von der Gesellschaft der Wissenschaften zu Göttingen, Mathematisch-Physikalische Klasse 1918, pp. 235 - 257.

Penchev, V. (2021 June 8) "The Symmetries of Quantum and Classical Information. The Resurrected "Ether" of Quantum Information," SSRN,

https://papers.ssrn.com/sol3/papers.cfm?abstract id=3861105

or https://dx.doi.org/10.2139/ssrn.3861105.

Penchev, V. (2021 February 2025) "Natural Cybernetics of Time, or about the Half of any Whole," SSRN, https://papers.ssrn.com/sol3/papers.cfm?abstract id=3750608 or https://dx.doi.org/10.2139/ssrn.3750608.

Penchev, V. (2020 October 20) "Two deductions: (1) from the totality to quantum information conservation; (2) from the latter to dark matter and dark energy," SSRN,

https://papers.ssrn.com/sol3/papers.cfm?abstract id $=3683658$ or

https://dx.doi.org/10.2139/ssrn.3683658.

Penchev, V. (2020 October 5) "Quantum-Information Conservation. The Problem About 'Hidden Variables', or the 'Conservation of Energy Conservation' in Quantum Mechanics: A Historical Lesson for Future Discoveries," SSRN, https://dx.doi.org/10.2139/ssrn.3675319 or

https://papers.ssrn.com/sol3/papers.cfm?abstract id=3675319 .

Penchev, V. (2020 August 25) "The Relationship of Arithmetic as Two Twin Peano Arithmetic(s) and Set Theory: a New Glance From the Theory of Information." SSRN,

https://papers.ssrn.com/sol3/papers.cfm?abstract id=3656179 or 
https://dx.doi.org/10.2139/ssrn.3656179 .

Penchev, V. (2020 July 10) "Quantum Information as the Information of Infinite Series," SSRN, https://papers.ssrn.com/sol3/papers.cfm?abstract id $=3630063$ or

https://dx.doi.org/10.2139/ssrn.3630063.

Penchev, V. (2020 July 1) "Skolem's 'Paradox' As Logic of Ground: The Mutual Foundation of Both Proper and Improper Interpretations," SSRN, https://dx.doi.org/10.2139/ssrn.3640109 or https://papers.ssrn.com/sol3/papers.cfm?abstract id=3640109.

Penchev, V. (2013) "The Kochen - Specker theorem in quantum mechanics: a philosophical comment," Philosophical Alternatives 22 (1): 67-77; 22 (3): 74-83,

https://philpapers.org/rec/PENTK-2 \& https://philpapers.org/rec/PENTK .

Penchev, V (2011) "The Paradox of Skolem and Quantum Information," Philosophical Alternatives 20 (2): 131-147 (in Bulgarian), https://philpapers.org/rec/PEN-23 .

Shannon, C. (1948) "A Mathematical Theory of Communication," The Bell System Technical Journal 27 (3): 379-423; 27 (4) 623-656.

Skolem, T. (1922) "Einige Bemerkungen zur axiomatischen Begründung der Mengenlehre," in: Matematikerkongressen $i$ Helsingrofs den 4-7 Juli 1922, Den femte skandinaviska matematikerkongressen. Redogörelse, Helsinki: Akademiska-Bokhandeln, pp. 217-232 (1923).

Whitehead, N.A. \& B. Russel (any edition) Principia Mathematica. Volume III.

Wiener, N. (1948) Cybernetics: Or Control and Communication in the Animal and the Machine. Cambridge (Mass.) MIT press.

Wiener, N. (1950) The Human use of human beings, cybernetics and society. Boston, Houghton Mifflin Co.

Wiener, N. (1964) God \& Golem, Inc.: A Comment on Certain Points Where Cybernetics Impinges on Religion. Cambridge (Mass.), MIT Press.

Zermelo, E. (1904) "Neuer Beweis, dass jede Menge Wohlordnung werden kann (Aus einem an Herrn Hilbert gerichteten Briefe)," Mathematische Annalen 59 (4): 514-516.

Zermelo, E. (1908) "Neuer Beweis für die Möglichkeit einer Wohlordnung," Mathematische Annalen 65 (1): 107-128. 